\title{
Sonic hedgehog pathway dysregulation in skin basal-cell carcinoma of a Polish population
}

\author{
Aleksandra Lesiak ${ }^{1}$, Dorota Sobolewska-Sztychny ${ }^{1}$, Marian Danilewicz ${ }^{2}$, \\ Michal Rogowski-Tylman ${ }^{1}$, Anna Sysa-Jedrzejowska ${ }^{1}$, Michal Sobjanek ${ }^{3}$, \\ Irmina Olejniczak-Staruch ${ }^{1}$, Joanna Narbutt ${ }^{1}$
}

\author{
${ }^{1}$ Department of Dermatology and Venereology, Medical University of Lodz, Poland \\ ${ }^{2}$ Department of Pathology, Medical University of Lodz, Poland \\ ${ }^{3}$ Department of Dermatology, Venereology, and Allergology, Medical University of Gdansk, Poland
}

\begin{abstract}
Sonic hedgehog (Shh) pathway impairment plays a key role in the pathogenesis of basal-cell carcinomas (BCC), the most frequent skin tumor among Caucasians. Shh, Smo, and Gli2 family proteins are necessary for adequate and controlled cell proliferation. The aim of this study was to evaluate Shh, Smo, and Smo expression in BCC skin biopsies taken from sun-exposed areas. 41 BCC skin biopsies and 22 healthy skin specimens (the control group) taken from the same areas served as material for the study. All specimens were immunohistochemically stained with monoclonal antibodies directed against the chosen proteins. Shh and Smo expression (cytoplasmic pattern) were recorded semiquantitatively using a four-grade score (0-3). Gli2 expression (nuclear pattern) was determined using an image analysis system (semiautomatic function). The immunoexpression of the Shh and Smo proteins significantly increased in the BCC group, as compared with the normal controls (for Shh, the mean intensity was 1.67 in BCC vs. 1.17 in the control group, $\mathrm{p}<0.001$; for Smo, the mean intensity was 1.46 in BCC vs. 0.99 in the control group, $\mathrm{p}<0.001$ ). The staining for Gli2 in the BCC group was completely negative, but indicated the presence of Gli2 in the control patients (1.15 Gli2 + cells/100 cells). Sonic hedgehog pathway dysregulation may play an important role in skin cancerogenesis leading to BCC development. (Folia Histochemica et Cytobiologica 2013, Vol. 51, No. 3, 219-224)
\end{abstract}

Key words: basal-cell carcinoma, Shh, Smo, Gli2, normal epidermis, ultraviolet radiation

\section{Introduction}

Basal-cell carcinoma (BCC) is the most frequent skin cancer among Caucasians, as well as the most common of all human cancers. Recently, there has been a significant increase in the incidence of BCC, especially among women [1]. BCC is generally a disorder of white individuals, arising particularly in sun-exposed body areas, most commonly on the head and neck, but also on the trunk and extremities [2].

Correspondence address: A. Lesiak,

Department of Dermatology Medical University of Lodz,

Krzemieniecka St. 5; 94-017 Lodz, Poland;

tel./fax: +48 $426867981,+48426884565$

e-mail: aleksandra.lesiak@umed.lodz.pl
The development of BCC is clearly associated with ionizing radiation, arsenic, and ultraviolet radiation, the last of which is the most important environmental risk factor. Epidemiological studies have shown that BCC risk correlates better with intermittent sun exposure than with cumulative lifetime sun exposure [3]. Short-wavelength UVB radiation (290-320 nm) has been shown to play the most significant role in BCC carcinogenesis. UVB radiation damages DNA and its repair system and alters the immune system, resulting in progressive genetic alterations and the formation of neoplasms. Experimental data indicate that a range of mutations is caused by UV radiation, one of which activates the hedgehog intercellular signaling pathway genes [4].

The hedgehog signaling pathway was first described in genetic studies of embryonic mutants of the fruit fly Drosophila melanogaster. Fruit flies have 
a single $\mathrm{HH}$ gene, whereas vertebrates have evolved three different types of homolog — the Sonic, Desert, and Indian types [4]. Among these, sonic hedgehog (Shh) is the most commonly expressed and the best characterized in embryonic and adult tissues $[5,6]$. Under physiological conditions, the sonic hedgehog pathway is responsible for normal embryogenesis, for regeneration of damaged tissues, and for regulation of cell proliferation [7]. Recent studies indicate that up-regulation of the Shh pathway may lead to the development of tumors in tissues of different origin-chiefly BCCs in the skin, medulloblastomas and gliomas in the brain, and rhabdosarcomas in muscle [8-10]. Mutations in the Shh signaling pathway are also involved in digestive tract, pancreatic, and smallcell lung cancers $[8,11-13]$.

Shh is synthesized as a $45 \mathrm{kDa}$ precursor, which activates the Shh signaling pathway by binding to the membrane receptor Patched (Ptch), which is a 12-pass transmembrane protein. After binding the sonic hedgehog protein Shh to the Ptch receptor, Smo protein is released and acts as transcriptional activator through the Gli nuclear factor, which moves from cytoplasm to nucleus. This results in controlled cell proliferation and differentiation through the activation of target genes.

It is important to note that $\mathrm{UV}$ radiation causes an inactivated mutation in the $P T C H$ gene. These events lead to dysregulation of the sonic hedgehog pathway by activating the Smo protein and the Gli transcriptional factors, which stimulate cell proliferation. In consequence, this may lead to the development of $\mathrm{BCC}$, for which UV radiation is the major risk factor.

The aim of this study was to assess the immunoexpression of the Shh, Smo, and Gli2 proteins in the nodular form of BCC localized on UV-exposed areas, compared with normal epidermis. Additionally, the correlations between the immunoexpression of Shh and Smo, as well as between Gli2 in the BCC group and the control group, were analyzed.

\section{Material and methods}

Patients. The study group consisted of 41 patients with BCC (mean age 59.2 years, $20 \mathrm{~F}, 21 \mathrm{M}$, phototype: I/II - 10, III - 23, IV - 8), diagnosed in the Department of Dermatology at the Medical University of Lodz between 2008 and 2010. All patients were Caucasian; none of them were organ transplant recipients, were being treated with immunosuppressive drugs, or suffered from any other malignancy. The patients were recruited at first attendance, when the biopsy for histology was taken. Skin biopsies for immunohistochemistry were taken during the second visit, when the diagnosis had been confirmed by histology (approximately
2 weeks after the first visit). Only patients with nodular BCC located in the upper cheek area were included. Additionally, 22 age- and sex-matched healthy subjects (mean age 55.3 years, $12 \mathrm{~F}, 10 \mathrm{M}$, phototype I/II - 4, III - 15, IV - 3) were enrolled to serve as a control group. Skin specimens in the control group were taken from the same areas as in the BCC group. Each subject gave written informed consent before entering the study, which had been approved by the local Ethics Committee. The subjects were assessed by a dermatologist for their skin phototype, according to the Fitzpatrick score, and for hair and eye color. The tissues were immediately snap-frozen in liquid nitrogen and stored at $-80^{\circ} \mathrm{C}$ until analysis.

Immunohistochemistry. Paraffin-embedded tissue sections were mounted onto SuperFrost slides, deparaffinized, and treated in a microwave oven in a solution of TRS (Target Retrieval Solution, Dako, Glostrup, Denmark) for 30 minutes $(2 \times 6$ minutes at $360 \mathrm{~W}, 2 \times 5$ at $180 \mathrm{~W}, 2 \times 4$ minutes at $90 \mathrm{~W}$ ), and were transferred to distilled water. Endogenous peroxidase activity was blocked using $0.3 \%$ hydrogen peroxide in distilled water for 30 minutes, and then sections were rinsed with Tris-buffered saline (TBS, Dako) and incubated for one hour with primary rabbit polyclonal antibody against Shh (ab 73958, dilution 1:100, Abcam, Cambridge, MA, USA), Smo (Abcam ab 72130, concentration $10 \mu \mathrm{g} / \mathrm{mL}$ ), and Gli2 (Abcam ab 26056; concentration $6 \mu \mathrm{g} / \mathrm{mL}$ ) proteins. Immunoreactive proteins were visualized using an EnVision horseradish peroxidase kit (Dako, Carpinteria, CA, USA), according to the instructions of the manufacturer. Visualization was performed by incubating the sections in a solution of 3,3'-diaminobenzidine (DakoCytomation, Glostrup, Denmark). After washing, the sections were counterstained with hematoxylin and coverslipped. For each antibody and for each sample, a negative control was processed. Negative controls were carried out by incubation in the absence of the primary antibody, and always yielded negative results.

In each specimen, the staining intensities of Shh and Smo were recorded semiquantitatively using two independent observers in 7-10 adjacent high-power fields, and graded from 0 (staining not detectable), 1 (weak immunostaining), 2 (moderate immunostaining intensity) and 3 (strong staining). The mean grade was calculated by averaging the grades assigned by two of the authors and approximating the arithmetical mean to the nearest unity.

Morphometry. Histological morphometry was performed using an image-analysis system consisting of a personal computer equipped with a Pentagram graphical tablet, an Indeo Fast card (frame grabber, true-color, real-time; Indeo, Taipei, Taiwan), and a color TV camera (Panasonic, Tokyo, Japan) coupled to a Carl Zeiss Jenaval microscope (Carl Zeiss, Jena, Germany). This system was programmed (MultiScan 8.08 software, Computer Scanning Systems, Warsaw, 
Poland) to calculate the number of objects (semiautomatic function), as has been previously described [14].

In brief, the colored microscopic images were saved serially in the memory of a computer, and the percentage of nuclei expressing the Gli2 antigen was estimated semi -automatically by counting 100 cells on ten monitor fields (0.0205 mm² each). Immunopositive cells were marked, so that in each case, 1000 cells were analyzed.

Statistical analysis. All values were expressed as mean \pm SD (standard deviation). The differences between groups were tested using Student's $t$-test for independent samples, preceded by evaluation of normality and homogeneity of variances using Levene's test. Additionally the Mann-Whitney $U$-test was employed where appropriate. Correlation coefficients were calculated using Spearman's method. The results were considered statistically significant when $\mathrm{p}<0.05$.

\section{Results}

In the basal-cell carcinoma (BCC) group, staining for Shh was positive in 34 of 41 patients; for Smo, in 32 of 41 participants. Both Shh and Smo proteins were detected in some, though not all, cancer cells (Figures 1A, 2A, 3A, and 4A) and, in a few cases, on inflammatory infiltrates. However, for the present study, the staining on inflammatory cells was not taken into consideration. In the controls, weak staining for Shh was positive in 16 of 22 cases; for Smo, in 14 of 22 specimens. The immunoexpression of Shh and Smo proteins was found in the keratinocytes (Figures 1B, 2B, 3B, and 4B) of normal epidermis. No reactivity was found in sebaceous glands for these proteins. Shh immunoexpression was detected in the cytoplasm, Smo was expressed in both the cytoplasm and cell membrane, and Gli2 immunoexpression was exclusively nuclear in epithelial cells. Sporadic Gli2 immunoexpression on inflammatory infiltrates was not taken into consideration. The semiquantitative and morphometric data for the immunoexpression of Shh, Smo, and Gli2 in BCC and the control group are given in Table 1. The immunoexpression of the Shh and Smo proteins was significantly increased in the $\mathrm{BCC}$ group, as compared to the normal controls. The staining for Gli2 in BCC group was completely negative, (Figures 5A and 6A) but Gli2 was detected in the control patients (Figures 5B and 6B).

The correlations between the immunoexpression of Shh and Smo, as well as between Gli2 in basal-cell carcinoma and the controls, are shown in Table 2. The immunoexpression of Shh was significantly positively correlated with the immunoexpression of Smo in both tested groups. In the controls, the immunoexpression of Shh tended to be positively correlated with staining for Gli2, but this correlation did not reach statistical significance.

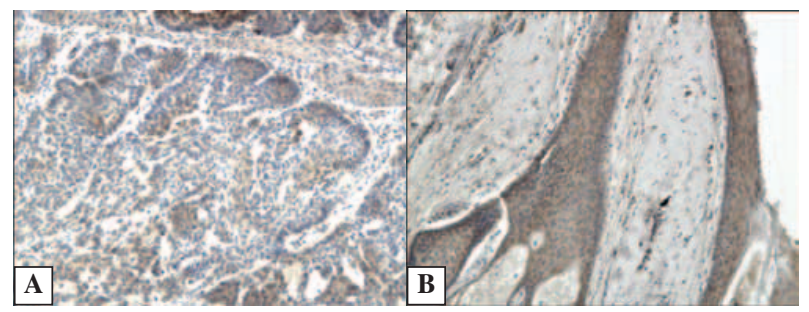

Figure 1. Immunoexpression of Shh in BCC cells (A) and in normal epidermis $(\mathbf{B}) . \times 100$

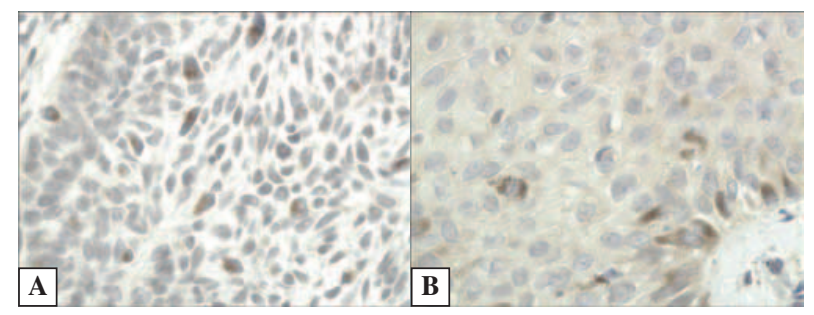

Figure 2. Immunoexpression of Shh in BCC cells (A) and in normal epidermis $(\mathbf{B}) . \times 100$

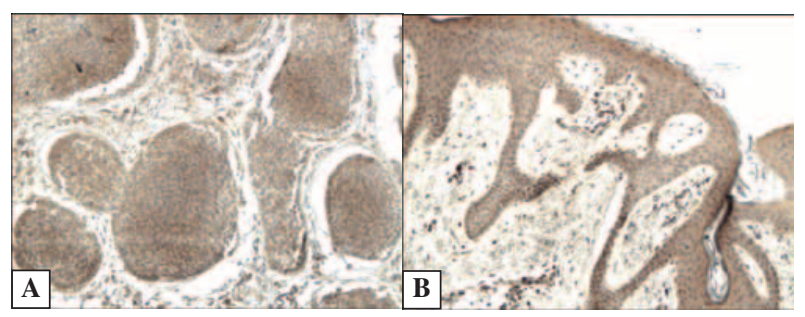

Figure 3. Immunoexpression of Smo in several BCC cells (A) and in normal epidermis (B). $\times 100$

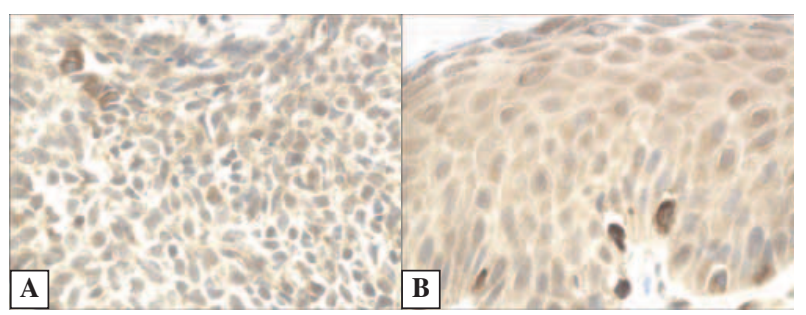

Figure 4. Immunoexpression of Smo in several BCC cells (A) and in normal epidermis (B). $\times 400$

\section{Discussion}

The pathogenesis of BCC development has been widely analyzed in recent years. Many scientific communications indicate that the pivotal molecular abnormality in BCC carcinogenesis is the inappropria- 
Table 1. A comparison of semiquantitative and quantitative data of Shh, Smo, and Gli2 immunoexpression in basal-cell carcinoma and controls

\begin{tabular}{|l|c|c|c|}
\hline & $\begin{array}{c}\text { Shh } \\
\text { (mean score) }\end{array}$ & $\begin{array}{c}\text { Smo } \\
\text { (mean score) }\end{array}$ & $\begin{array}{c}\text { Gli2 } \\
\text { (\%) }\end{array}$ \\
\hline $\begin{array}{l}\text { Basal-cell } \\
\text { carcinoma } \\
(\mathrm{n}=41)\end{array}$ & $1.67 \pm 0.48$ & $1.46 \pm 0.4$ & $0.00 \pm 0.00$ \\
\hline $\begin{array}{l}\text { Control group } \\
(\mathrm{n}=22)\end{array}$ & $1.17 \pm 0.62$ & $0.99 \pm 0.39$ & $1.15 \pm 0.66$ \\
\hline P value & $\mathrm{p}<0.001$ & $\mathrm{p}<0.001$ & $\mathrm{p}=0.000$ \\
\hline
\end{tabular}

The immunoreactivity score for Shh and Smo proteins was obtained as described in Methods. Data present mean \pm SD. The percentage of cells expressing the Gli2 immunoreactivity was measured as described in Methods

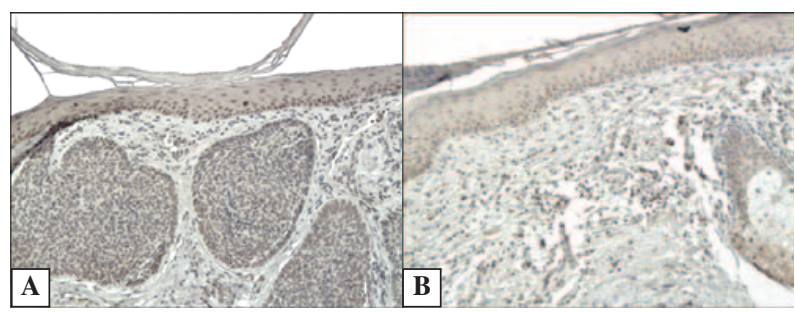

Figure 5. Negative Gli2 staining in BCC (A), nuclear staining for Gli2 in normal epidermis $(\mathbf{B}) . \times 100$

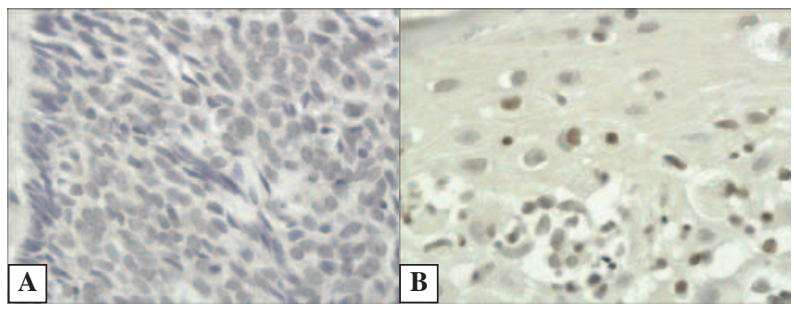

Figure 6. Negative Gli2 staining in BCC (A), nuclear staining for Gli2 in normal epidermis $(\mathbf{B}) . \times 400$

Table 2. Correlations between immunoexpression of Shh, Smo, and Gli2 in basal-cell carcinoma and controls

\begin{tabular}{|l|c|c|}
\hline Pair of variables & \multicolumn{1}{|c|}{$\begin{array}{c}\text { Basal-cell } \\
\text { carcinoma } \\
(\mathbf{n}=\mathbf{4 1})\end{array}$} & $\begin{array}{c}\text { Control group } \\
(\mathbf{n}=\mathbf{2 2})\end{array}$ \\
\hline Shh vs. SMO & $\mathrm{r}=0.49, \mathrm{p}<0.001$ & $\mathrm{r}=0.68, \mathrm{p}<0.02$ \\
\hline Shh vs. Gli2 & - & $\mathrm{r}=0.55, \mathrm{p}=0.06(\mathrm{NS})$ \\
\hline
\end{tabular}

Statistical analysis was performed as described in Methods

te activation of the Shh signaling pathway. Ling et al. [15], as well as Xie et al. [16], have reported that more than $70 \%$ of sporadic BCCs have detectable genetic mutations in components of the Shh signaling pathway.
In our study, we used the immunohistochemical method to analyze the expression of Shh, Smo, and Gli2 proteins and their alteration in the pathogenesis of the nodular type of BCC, which is usually located on UV-exposed body areas. We observed significantly increased immunoexpression of the Shh and Smo proteins in the BCC group, as compared to normal controls. Furthermore, the immunoexpression of Shh was significantly positively correlated with the immunoexpression of Smo in both tested groups. This observation testifies to the interaction between them.

In 1999, Michiko et al. [17] published a study describing the overexpression of Smo mRNA in BCC compared with its expression in normal human skin. In addition, they observed that Smo mRNA expression might be associated with the progression of $\mathrm{BCC}$ tumors, and divided BCCs into two histologic types: superficial and nodular. We analyzed the nodular type of BCC localized on the face, and our results are in line with those of other authors. The study by Xie et al. [16] showed direct evidence that mutated Smo can function as an oncogene in BCCs, as they observed skin abnormalities similar to BCCs that developed in transgenic murine skin overexpressing mutant Smo.

Many studies also indicate high levels of ultraviolet-specific mutations in the $S M O$ gene in groups of BCC patients, which confirms its significance in the development of BCC, probably by leading to constitutive activation of the protein. Couvé-Privat et al. [18] have analyzed the involvement of the $S M O$ gene in the genesis of BCC by examining skin tumors from patients with xeroderma pigmentosum. On the basis of this analysis, they indicated the presence of relatively high levels of Smo proto-oncogene mutations in BCCs. Our results also support this hypothesis.

The significance of the Shh protein in cancerogenesis was confirmed in the study of Fan and Khavari [19], who indicated that the cell-cycle withdrawal normally associated with terminal differentiation is blocked in human keratinocytes that overexpress Shh. This finding may support our results.

It is also known that Shh induces features of basal keratinocytes into $\mathrm{HaCaT}$ organotypic cell cultures. Bigelow et al. [20] have demonstrated that Shh in $\mathrm{HaCaT}$ keratinocytes grown in organotypic cultures induced a basal-cell phenotype, as proven by their morphology, transepithelial staining of cytokeratin, and suprabasilar proliferation. Furthermore, Shh also induced keratinocyte infiltration in the underlying collagen matrix.

Deregulation of the Shh pathway is associated with basal-cell carcinoma (BCC) due to up-regulation of the GLI genes (GLII and GLI2). The significance of the role of the GLI genes in mediating carcinogene- 
sis in different structures has been shown by many authors.

To date, there are individual data which have led to inconsistent results in regard to Gli2 expression in skin BCC, perhaps demonstrating variability in the selection and numbers of patients and controls, or else reflecting the differences of in vivo and in vitro studies and results from different populations. In our study, the staining for Gli2 in the BCC group was completely negative, but Gli2 was detected in the control patients. On the contrary, a study by Ikram et al. [21] has shown that Gli2 is expressed in normal skin, as well as in BCC tumor islands. In 2012, Agyeman et al. [22] published a study describing the importance of targeting Gli downstream of Smo in the induction of cell death in human colon carcinoma cells. The human GLI genes have also been shown to act as oncogenes in brain tumors, for example medulloblastomas [23]. More recent studies have also concentrated on the correlation between the GLII and GLI2 genes. Numerous studies indicate a positive feedback loop between these, which reveals that Gli1 may be a direct target of Gli2. In our study, we did not examine these relationships, but we additionally analyzed the correlation between Shh and Gli2 in the BCC and control groups. We observed in controls that the immunoexpression of Shh tended to be positively correlated with staining for Gli2, but this correlation did not obtain statistical significance.

Many scientific communications indicate the significance of the overexpression of hedgehog signaling molecules and their involvement in different types of cancer. For example, Tao et al. [24] confirmed that Shh signaling is involved in breast ductal changes and malignant transformation in triple-negative breast cancer. One study has demonstrated that ectopic overexpression of Shh induces stromal expansion and metaplasia in the adult murine pancreas [25]. On the base of laboratory and clinical investigations, and our own observations, we can conclude that the investigated signaling pathway plays a key role in the development of many types of cancer - especially in the most common human cancer in the fair-skinned population, the nodular type of BCC.

Pharmacological inhibition of Smo or of downstream effectors of this pathway could provide an effective treatment for BCCs, and perhaps for other cancers as well. The discovery of small-molecule antagonists of Shh signaling, such as cyclopamine, has opened up exciting new prospects for BCC therapy [26-28]. Recent data indicates the role of cyclopamine in blocking the growth of UV-induced BCCs in mice. Additionally, it is known that this molecule may also inhibit the proliferation of BCC murine cell lines [2]. One clinical report concerning four patients with BCC described the rapid clinical regression of the cancer in response to topical cyclopamine versus placebo [29]. Other histological and immunohistochemical analysis have reported apoptosis and increased markers of differentiation in response to Shh inhibition [30].

In other studies, the potential role of various substances has been shown, offering new therapeutic targets for the treatment of BCCs. The authors suggest that the possible role of sterols and vitamin $\mathrm{D}$ in Shh signaling be investigated, but more work needs to be done to confirm these results [30].

On the basis of our analysis, we observed significantly increased immunoexpression of Shh and Smo proteins in the BCC group, compared with the normal controls. This may be evidence of the significance of the Shh pathway in the development of BCC.

These results allow use to conclude that sonic hedgehog pathway dysregulation may play a significant role in skin cancerogenesis, leading to BCC development. To our knowledge, the expression of the sonic hedgehog pathway in BCCs has not been evaluated previously in the Polish population, and this pathway was considerably up-regulated in the BCCs, compared with healthy skin. Moreover, many other studies that examined BCCs were self-reported, and did not record the body site, while in our study, the diagnosis of $\mathrm{BCC}$ was confirmed by pathology, and only those subjects with BCCs on the upper cheek (a site of high solar exposure) were analyzed. A greater understanding of the role of the Shh signaling pathway impairment in this population may lead to an effective strategy for the prevention of $\mathrm{BCC}$ development among the general population.

\section{Acknowledgment}

The study was funded by grants from the Medical University of Lodz (no. 503/1-152-01/503-01) and from the Polish Scientific Committee (no. NN402474731). The authors declare no conflict of interest.

\section{References}

1. Lesiak A, Slowik-Rylska M, Rogowski-Tylman M et al. Risk factors in Central Poland for the development of superficial and nodular basal cell carcinomas. Arch Med Sci. 2010;6: 270-275.

2. Tang JY, So P, Epstein EH Jr. Novel Hedgehog pathway targets against Basal Cell Carcinoma. Toxicol Appl Pharmacol. 2007;224:257-264.

3. Corona R, Dogliotti E, D'Errico M et al. Risk factors for basal cell carcinoma in a Mediterranean population: role of recreational sun exposure early in life. Arch Dermatol. 2001;137:1162-1168.

4. Omar L. Correlations between the Sonic Hedgehog Pathway and basal cell carcinoma. Int J Dermatol. 2007; 46:1113-1117. 
5. Ruiz IA., Sanchez P, Dahmane N. Gli and hedgehog in cancer: tumours, embryos and stem cells. Nat Rev Cancer. 2002;2:361-372.

6. Ruiz IA, Palma V, Dahmane N. Hedgehog — Gli signalling and the growth of the brain. Nat Rev Neurosci. 2002;3:24-33.

7. Lupi O. Correlations between the Sonic Hedgehog pathway and basal cell carcinoma. Int J Dermatol. 2007;46:1113-1117.

8. Daya-Grosjean L, Couvé-Privat S. Sonic hedgehog signaling in basal cell carcinomas. Cancer Let. 2005;225:181-192.

9. Mullor JL, Sanchez P, Altaba AR. Pathways and consequences: hedgehog signaling in human disease. Trends Cell Biol. 2002;12:562-569

10. Wetmore C. Sonic hedgehog in normal and neoplastic proliferation: insight gained from human tumors and animal models. Curr Opin Genet Dev. 2003;13:34-42.

11. Berman DM, Karhadkar SS, Maitra A et al. Widespread requirement for Hedgehog ligand stimulation in growth of digestive tract tumours. Nature. 2003;425:846-851.

12. Thayer SP, di Magliano MP, Heiser PW et al. Hedgehog is an early and late mediator of pancreatic cancer tumorigenesis. Nature. 2003;425:851-856.

13. Watkins DN, Berman DM, Burkholder SG et al. Hedgehog signalling within airway epithelial progenitors and in small-cell lung cancer. Nature. 2003;422:313-317.

14. Stasikowska-Kanicka O, Wagrowska-Danilewicz M, Białek I et al. The immunoexpression of Shh, Smo and Gli2 in Helicobacter pylori positive and negative gastric biopsies. Pol J Pathol 2012;1:25-30.

15. Ling G, Ahmadian A, Persson A et al. PATCHED and p53 gene alterations in sporadic and hereditary basal cell cancer. Oncogene. 2001;20:7770-7778.

16. Xie J, Murone M, Luoh SM et al. Activating Smoothened mutations in sporadic basal-cell carcinoma. Nature. 1998;391:90-92.

17. Tojo M, Mori T, Kiyosawa $\mathrm{H}$ et al. Expression of sonic hedgehog signal transducers, patched and smoothened, in human basal cell carcinoma. Path Intern. 1999;8:687-694.

18. Couvé-Privat S, Bouadjar B, Avril MF et al. significantly high levels of ultraviolet-specific mutations in the smoothened gene in basal cell carcinomas from DNA repair-deficient xeroderma pigmentosum patients. Cancer Res. 2002;62:7186-7189.
19. Fan H, Oro AE, Scott MP et al. Induction of basal cell carcinoma features in transgenic human skin expressing Sonic Hedgehog. Nat Med. 1997;3:788-792.

20. Bigelow R, Jen EY, Delehedde M et al. Sonic hedgehog induces epidermal growth factor dependent matrix infiltration in HaCa T keratinocytes. J Invest Dermatol. 2005;124:457-465.

21. Ikram MS, Neill GW, Regl G et al. GLI2 is expressed in normal human epidermis and BCC and induces GLI1 expression by binding to its promoter. J Invest Dermatol. 2004;122: 1503-1509.

22. Agyeman A, Mazumdar T, Houghton JA. Regulation of DNA damage following termination of hedgehog $(\mathrm{HH})$ survival signaling at the level of the GLI genes in human Colon Cancer. Oncotarget. 2012;3:851-865.

23. Kinzler KW, Bigner SH, Bigner DD et al. Identification of an amplified, highly expressed gene in a human glioma. Science. 1987;236:70-73.

24. Tao Y, Mao J, Zhang Q et al. Overexpression of Hedgehog signaling molecules and its involvement in triple-negative breast cancer. Oncol Lett. 2011;2:995-1001.

25. Fendrich V, Oh E, Bang S et al. Ectopic Overexpression of sonic hedgehog (Shh) induces stromal expansion and metaplasia in the adult murine pancreas. Neoplasia. 2011;13: 923-930.

26. Taipale J, Chen JK, Cooper MK et al. Effects of oncogenic mutations in smoothened and patched can be reversed by cyclopamine. Nature. 2000;406:1005-1009.

27. Chen JK, Taipale J, Young KE et al. Small molecule modulation of smoothened activity. Proc Natl Acad Sci USA. 2002;99:14071-14076.

28. Williams JA, Guicherit OM, Zaharian BI et al. Identification of a small molecule inhibitor of the hedgehog signaling pathway: effects on basal cell carcinoma-like lesions. Proc Natl Acad Sci USA. 2003;4:4616-4621.

29. Lin TL, Matsui W. Hedgehog pathway as a drug target: Smoothened inhibitors in development. Onco Targets Ther. 2012;5:47-58.

30. Tabs S, Avci O. Induction of the differentiation and apoptosis of tumor cells in vivo with efficiency and selectivity. Eur $J$ Dermatol. 2004;14:96-102. 